\title{
A Research of Achromatic and B-Chromatic Number of Multistar-Related Graphs
}

\author{
K.P.Thilagavathy, A.Santha, K.Paramasivam
}

\begin{abstract}
The structural properties, achromatic number and b-chromatic number of central graph of double star graph and triple star graph have been studied in [6]. In this paper, these studies have been carried out, for the central graph of any multi star graph and central graph of shadow graph of star graph and double star graph.

Key Words - Double Star graph, Triple Star graph, multi star graph, achromatic number, b-chromatic number, Shadow graph Mathematics subject classification: $05 \mathrm{C} 15$
\end{abstract}

\section{INTRODUCTION}

Graph theory and graph colouring have wide applications in physics, chemistry, social sciences, networking, VLSI circuits [ 4 ] and many other fields.

A Star graph $a_{n}$ is the bi partite graph $K_{1, n}$. Themultistar graph $K_{m}\left(a_{n_{1}}, a_{n_{2}}, \ldots, a_{n_{m}}\right)$ is the graph got by adding $n_{1}, n_{2}, \ldots . . n_{m}$ leaves respectively to the $m$ vertices of the complete graph $K_{m}$. The number of spanning trees of a specific family of multistar graph was studied by Nikolopoulous and Rondogiannis [3].

Consider a simple undirected graph $G$. To form its central graph $C(G)$ we introduce a new node on every edge of $G$ and join the non-adjacent nodes of $G$.In 1999, Irving and Manlove introduced the idea of b-chromatic number, which is the maximum value of $k$ for which $G$ has a proper colouring with $k$ colours in such a way that every colour is adjacent to every other colour.

The achromatic number was introduced by Harary[1].An achromatic colouring is a proper vertex colouring such that each pair of colours is adjacent by at least one edge. The largest possible number of colours in an achromatic colouring of $G$ is called the achromatic number of $G$ and it is denoted by $\psi(G)$.

The concept of generalisation of achromatic colouring of graphs was studied by Roopesh and Thilagavathi[5], who proved that the achromatic number of the central graph of any simple graph $G(V, E)$ with $|V|=n$ is less than or equal to $n+1$. The achromatic and b- chromatic number of various graphs have been studied in $[6,7,8]$

The Shadow graph $D_{2}(G)$, of a connected graph $G$ is constructed by taking two copies of $G$, say $G^{\prime}$ and $G^{\prime \prime}$.Join

Revised Manuscript Received on August 14, 2019.

K.P.Thilagavathy, Assistant Professor, Department of Science and Humanities, Kumaraguru College of Technology, Coimbatore-641049. T.N. India

A.Santha, Associate Professor, Department of Science and Humanities, Kumaraguru College of Technology, Coimbatore-641049. T.N. India

K.Paramasivam, Professor, Department of Electrical and Electronics Engineering, Kumaraguru College of Technology, Coimbatore-641049 T.N. India each vertex $u^{\prime}$ in $G^{\prime}$ to the neighbours of the corresponding vertex $v^{\prime}$ in $G^{\prime \prime}$.The following results have been discussed in [8].

The Structural Properties and the colouring of Central graph of Double Star Graph

1. The number of vertices in the graph $C\left[K_{2}\left(a_{n}, a_{r}\right)\right]$ is $p=2(n+r)+3$

2. The number of edgesin the graph $C\left[K_{2}\left(a_{n}, a_{r}\right)\right]$ is $q=\frac{(n+r+1)(n+r+4)}{2}$

3. The maximum degree in the graph $C\left[K_{2}\left(a_{n}, a_{r}\right)\right]$ is $\Delta=n+r+1$

4. For any Double Star graph $K_{2}\left(a_{n}, a_{r}\right)$ the achromatic number $\psi\left[C\left(K_{2}\left(a_{n}, a_{r}\right)\right)\right]=n+r+2$

5. For any Double Star graph $K_{2}\left(a_{n}, a_{r}\right)$ the b-chromatic number $\varphi\left[C\left(K_{2}\left(a_{n}, a_{r}\right)\right)\right]=n+r+1$

The Structural Properties and the colouring of Central graph of Triple Star Graph

1. The number of vertices in the graph $C\left[K_{3}\left(a_{n}, a_{r}, a_{t}\right)\right]$ is $p=2(n+r+t+3)$

2. The number of edgesinthe graph $C\left[K_{3}\left(a_{n}, a_{r}, a_{t}\right)\right]$ is $q=\frac{(n+r+t+3)(n+r+t+4)}{2}$

3. The maximum degree in the graph $C\left[K_{3}\left(a_{n}, a_{r}, a_{t}\right)\right]$ is $\Delta=n+r+t+2$

4. For any Triple Star graph $K_{3}\left(a_{n}, a_{r}, a_{t}\right)$ the bchromatic number $\varphi\left[C\left(K_{3}\left(a_{n}, a_{r}, a_{t}\right)\right)\right]=n+r+$ $t+1$

5. For any Triple Star graph $K_{3}\left(a_{n}, a_{r}, a_{t}\right)$ the achromatic number $\psi\left[C\left(K_{3}\left(a_{n}, a_{r}, a_{t}\right)\right)\right]=n+r+$ $t+3$

The generalisation of the above results to multi star graphs is discussed here.

The Achromatic and b-chromatic number of Central graph of Multi Star graph

\section{Theorem 2.1}

The achromatic number of central graph of multi star graph is $\psi\left[C\left(K_{m}\left(a_{n_{1}}, a_{n_{2}}, \cdots, a_{n_{m}}\right)\right)\right]=p$, where $p$ denotes the number of vertices in the multi star graph.

Proof:

Let $K_{m}\left(a_{n_{1}}, a_{n_{2}}, \ldots, a_{n_{m}}\right)$ be a multi star graph formed by adding $n_{1}, n_{2}, \ldots, n_{m}$ leaves to the $m$ nodes of $K_{m}$. Let $p$ be the total number of vertices in the multi star graph. That is $p=n_{1}+n_{2}+\cdots+n_{m}+$

$m$.

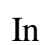

Published By: 
$C\left(K_{m}\left(a_{n_{1}}, a_{n_{2}}, \ldots, a_{n_{m}}\right)\right)$, the $p-m$ leaf vertices are given $p-m$ different colours and the remaining $m$ vertices of the complete graph are coloured by $m$ other colours. Hence $(p-m)+m$ colours are needed to colour this graph and by this construction it is the maximal one, and it is achromatic.

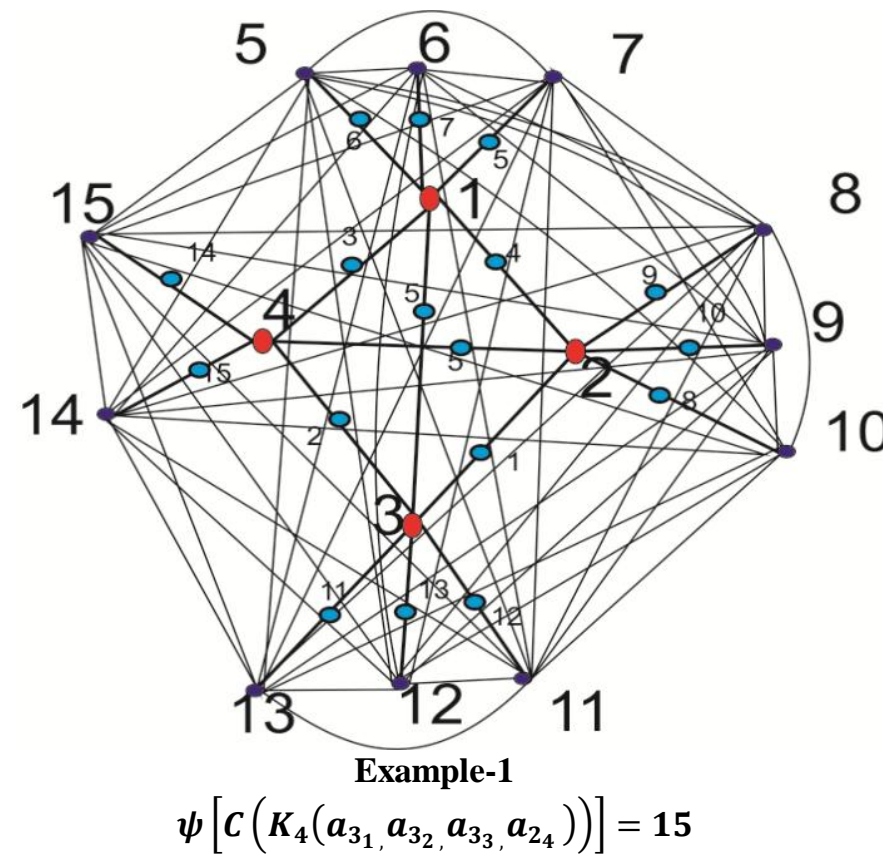

\section{RESULTS}

\section{Theorem 2.2}

The b-chromatic number of central graph of multi star graph

$\varphi\left[C\left(K_{m}\left(a_{n_{1}}, a_{n_{2}}, \ldots, a_{n_{m}}\right)\right)\right]=\left\{\begin{array}{l}p-\left(\frac{m}{2}\right), m=\text { even } \\ p-\left(\frac{m+1}{2}\right), m=\text { odd }\end{array}\right.$

where $p$ denotes the number of vertices in the multi star graph.

Proof:

Let $K_{m}\left(a_{n_{1}}, a_{n_{2}}, \ldots, a_{n_{m}}\right)$ be a multi star graph formed by adding $n_{1}, n_{2}, \ldots, n_{m}$ leaves to the $m$ nodes of $K_{m}$. Let $p$ be the total number of vertices in the multi star graph. That is $p=n_{1}+n_{2}+\cdots+n_{m}+m$.In $C\left[K_{m}\left(a_{n_{1}}, a_{n_{2}}, \cdots, a_{n_{m}}\right)\right]$ we need $p-m$ colours to colour the leaf vertices of $a_{n_{1}}, a_{n_{2}}, \ldots, a_{n_{m}}$. Then the complete graph $K_{m}$ can be coloured by $\frac{m}{2}$ colours if $m$ is even. The same colour can be given to two adjacent vertices. In the case when $m$ is odd we use $\frac{m+1}{2}$ colours to colour $m+1$ vertices of $K_{m}$ as in the above method. The last vertex can be coloured using any of the $\frac{m+1}{2}$ colours. This colouring is achromatic and it is the maximal one.

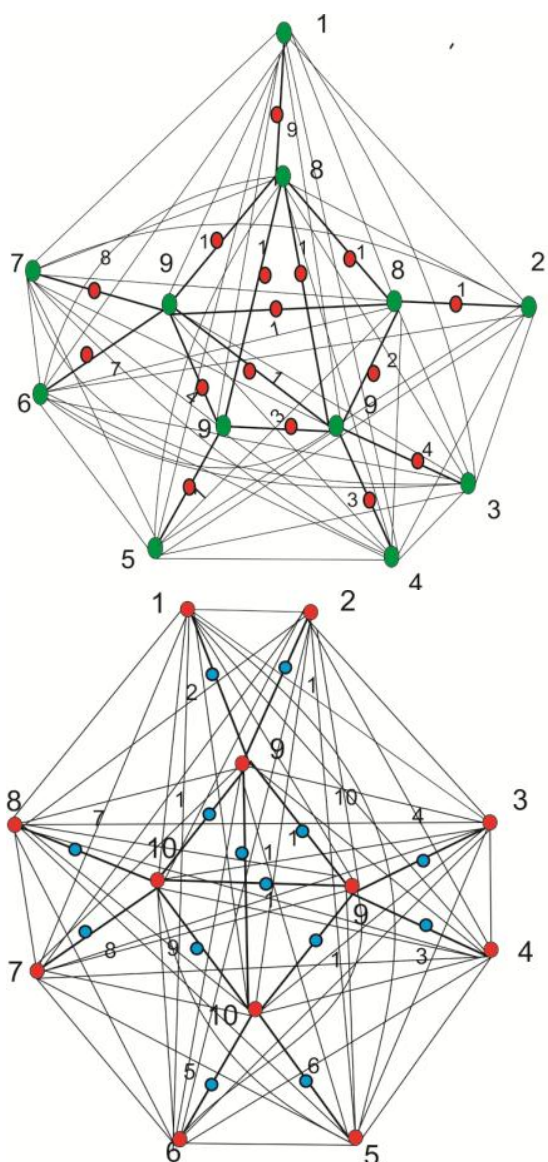

Example-2 Example-3

$$
\begin{gathered}
\varphi\left[C\left(K_{5}\left(a_{1_{1}}, a_{2_{1}}, a_{3_{2}}, a_{4_{2}}, a_{5_{2}}\right)\right)\right]=9 \\
\varphi\left[C\left(K_{4}\left(a_{1_{2}}, a_{2_{2}}, a_{3_{2}}, a_{4_{2}}\right)\right)\right]=10
\end{gathered}
$$

3. The Achromatic and b-chromatic number of Central graph of Shadow graph of Star graphs

The Structural properties of central graph of Shadow graph of Star graphs

1. The number of vertices in the graph $C\left(D_{2}\left(K_{1, n}\right)\right)$ is $p=6 n+2$

2. The number of edges in the graph $C\left(D_{2}\left(K_{1, n}\right)\right)$ is $q=$ $2 n^{2}+7 n+1$

3. The maximum degree in the graph $C\left(D_{2}\left(K_{1, n}\right)\right)$ is $\Delta=2 n+1$

\section{Theorem3.1}

The achromatic number of central graph of $D_{2}\left(K_{1, n}\right)$ is $\psi\left[C\left(D_{2}\left(K_{1, n}\right)\right)\right]=2(n+1)$

Proof: Let $K_{1, n}$ be the star graph. In $D_{2}\left(K_{1, n}\right)$, let $v_{0}, v_{1}, \ldots, v_{n}$ be the vertices of the first star graph and $u_{0}, u_{1}, \ldots, u_{n}$ be the corresponding vertices of the second star graph. By the definition of the shadow graph all the $v_{i}{ }^{\prime} s$ are connected with $u_{0}$ and all the $u_{i}{ }^{\prime} s$ are connected with $v_{0}$. In $C\left(D_{2}\left(K_{1, n}\right)\right)$, subdivide all the edges of $D_{2}\left(K_{1, n}\right)$ once and join all the non-adjacent vertices.

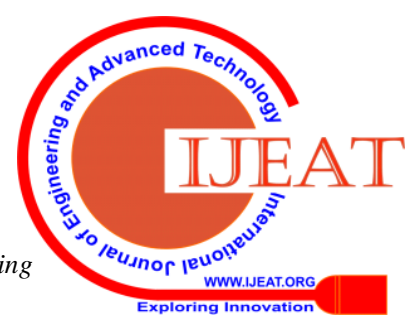


Let $u_{0} v_{i}$ be the newly introduced vertex on the edge joining the vertices $u_{0}$ and $v_{i}$ and $v_{0} u_{i}$ be the vertex on the edge joining the vertices $v_{0}$ and $u_{i}$ where $i=1,2,3 \ldots n$. Let $v_{0} v_{i}$ and $u_{0} u_{i}$ be the newly introduced vertices on the edges joining the vertices $v_{0}, v_{i}$ and $u_{0}, u_{i}$ respectively where $i=1,2,3 \ldots n$. Consider the two sets of colours $C=$ $\left\{C_{0}, C_{1}, \ldots, C_{n}\right\}$ and $C^{\prime}=\left\{C_{0}^{\prime}, C_{1}^{\prime}, \ldots, C_{n}^{\prime}\right\}$. Assign $C_{i}$ to $v_{i}$ and $C_{i}{ }^{\prime}$ to $u_{i}$ where $i=0,1,2,3 \ldots n$.

Hence the pairs of colours $\left(C_{i}, C_{j}\right)$ and $\left(C_{i}^{\prime}, C_{j}^{\prime}\right)$ where $i \neq j, j \neq 0$ are adjacent. The pair $\left(C_{0}, C_{0}^{\prime}\right)$ is adjacent. For making the remaining pairs of colours adjacent for an achromatic colouring, consider the following procedure: For $1 \leq i \leq n$ assign $\quad C_{0}$ to the vertices $u_{0} v_{i}$ and $u_{0} u_{i}$. For $1 \leq i \leq n$ assign $C_{0}^{\prime}$ to the vertices $v_{0} v_{i}$ and $v_{0} u_{i}$. By this construction any pair of colours in $C \cup C^{\prime}$ is adjacent by at least one edge and this colouring is the maximal possible one. Hence $\psi\left[C\left(D_{2}\left(K_{1, n}\right)\right)\right]=2(n+1)$.

Theorem 3.2

The b-chromatic number of central graph of $D_{2}\left(K_{1, n}\right)$ is $\varphi\left[C\left(D_{2}\left(K_{1, n}\right)\right)\right]=2 n+1$.

Proof: Let $K_{1, n}$ be the star graph. In $D_{2}\left(K_{1, n}\right)$, let $v_{0}, v_{1}, \ldots, v_{n}$ be the vertices of the first star graph and $u_{0}, u_{1}, \ldots, u_{n}$ be the corresponding vertices of the second star graph. By the definition of the shadow graph all the $v_{i}^{\prime} s$ are connected with $u_{0}$ and all the $u_{i}^{\prime} s$ are connected with $v_{0}$. In $C\left(D_{2}\left(K_{1, n}\right)\right)$, subdivide all the edges of $D_{2}\left(K_{1, n}\right)$ once and join all the non-adjacent vertices.

Let $u_{0} v_{i}$ be the newly introduced vertex on the edge joining the vertices $u_{0}$ and $v_{i}$ and $v_{0} u_{i}$ be the vertex on the edge joining the vertices $v_{0}$ and $u_{i}$ where $i=1,2,3 \ldots n$. Let $v_{0} v_{i}$ and $u_{0} u_{i}$ be the newly introduced vertices on the edges joining the vertices $v_{0}, v_{i}$ and $u_{0}, u_{i}$ respectively where $i=0,1,2,3 \ldots n$.

Consider $v_{i}, i=1,2,3, \ldots, n$. In $\quad C\left(D_{2}\left(K_{1, n}\right)\right), v_{i}$ is adjacent to all other $v_{j}{ }^{\prime} s,(j \neq 0)$ and all $u_{i}{ }^{\prime} s$. Similarly $u_{i}, i=1,2,3, \ldots, n$ is adjacent to all other $u_{j}{ }^{\prime} s,(j \neq 0)$ and $v_{i}{ }^{\prime} s, i=1,2,3 \ldots, n$.Also $v_{0}$ is adjacent only to $u_{0}$ and $u_{0}$ is adjacent only to $v_{0}$. Consider the two sets of colours $C=\left\{C_{0}, C_{1}, \ldots, C_{n}\right\}$ and $C^{\prime}=\left\{C_{1}{ }^{\prime}, \ldots, C_{n}{ }^{\prime}\right\}$.To make the colouring as b-chromatic consider the following colouring procedure:

- For $1 \leq i \leq n$ assign $C_{i}^{\prime}$ to the vertices $v_{0} v_{i}$

- For $1 \leq i \leq n$ assign $C_{i}$ to the vertices $v_{0} u_{i}$

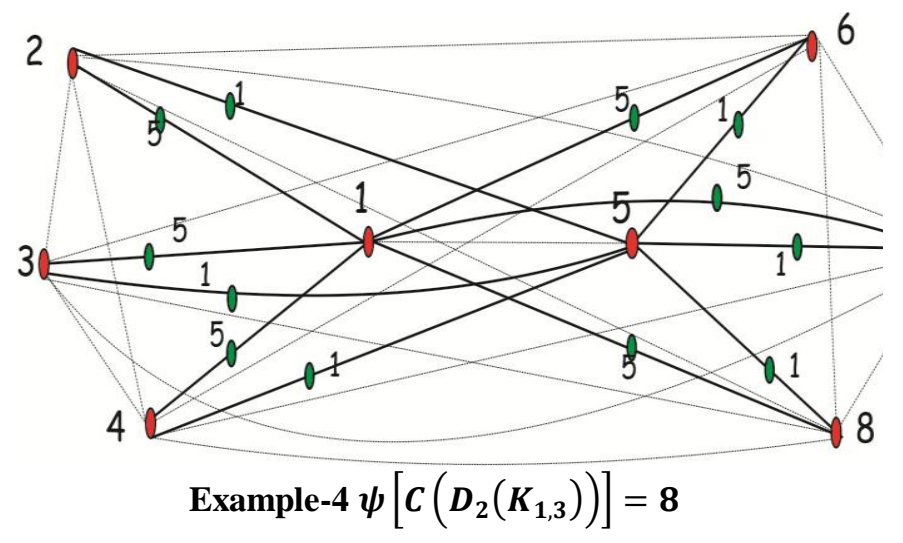

- For $1 \leq i \leq n$ assign $C_{0}$ to the vertices $u_{0} v_{i}$ and assign $C_{0}$ to the vertex $u_{0} u_{1}$

Here the representative vertices of the $2 n+1$ colour classes that are adjacent to a vertex in every other class are $v_{0}, v_{1}, \ldots, v_{n}, u_{1}, \ldots, u_{n}$.

$$
\text { Hence } \varphi\left[C\left(D_{2}\left(K_{1, n}\right)\right)\right]=2 n+1 \text {. }
$$

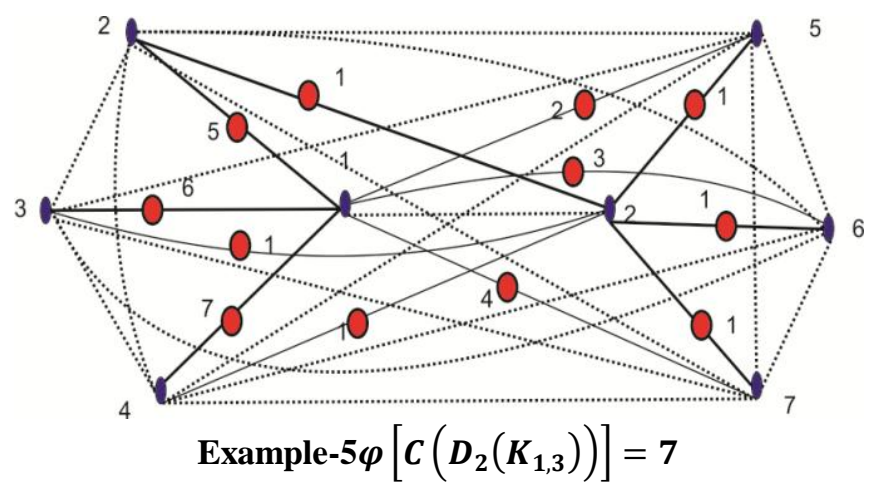

4. The Achromatic and b-chromatic number of Central graph of Shadow graph of Double Star graph

The Structural properties of central graph of Shadow graph of Double Star graph

1. The number of vertices in $D_{2}\left(K_{2}\left(a_{n}, a_{r}\right)\right)$ is $p=$ $2(2+n+r)$

2. The number of edges in $D_{2}\left(K_{2}\left(a_{n}, a_{r}\right)\right)$ is $q=$ $4(n+r)+2$

\section{Observation 4.1}

1. The achromatic number of central graph of $D_{2}\left(K_{2}\left(a_{n}, a_{r}\right)\right)$ is $\quad \psi\left[C\left(D_{2}\left(K_{2}\left(a_{n}, a_{r}\right)\right)\right)\right]=2(n+$ $r+2)$

2. The b-chromatic number of central graph of $D_{2}\left(K_{2}\left(a_{n}, a_{r}\right)\right)$ is $\varphi\left[C\left(D_{2}\left(K_{2}\left(a_{n}, a_{r}\right)\right)\right)\right]=2(n+$ r)

\section{CONCLUSION}

In this research work, the achromatic and b-chromatic numbers of central graph of double star, triple star and any multi star graph have been discussed.Also,the structural properties, the achromatic and b-chromatic numbers of the central graphsof shadow graph of star graph and double star graph have been studied.

\section{REFERENCES}

1. Harary, F. Graph Theory, Narosa Publishing 1969.

2. Irving, R.W and Manlove, D.F. "The b-Chromatic number of a graph", Discrete Applied Mathematics 91(1-3) 127-141, 1999.

3. Nikipoulos,S.D and Rondogiannis ,P. "On the Number of Spanning trees of Multistar Related Graphs", Volume 65,Issue 4,183-188,1998.

4. Paramasivam.K, Gunavathi. K,Sathishkumar.P, "Graph Theory Based Approach for Low Power Combinational Circuit Testing", proceedings of International AMSE conference MS2004, Lyon, France, July 5-6, 2004. 
5. Thilagavathi, K, Roopesh, N. 'Generalisationof achromatic colouring of central graphs', Electronic Notes in Discrete Mathematics, vol. 33, pp. 147-152, 2009.

6. Thilagavathy, K.P, Santha, A. 'A Note on Achromatic and bchromatic number of graphs', International Journal of Applied Mathematics and Statistics, 53(1),104-110, 2016.

7. Thilagavathy, K.P, Santha, A. 'An Achromatic Number and bchromatic number of Some Star related graphs'Proceedings of National conference on Developing Scenario in Applied Sciences and Communicative English, Kumaraguru College of Technology, 305-307, 2016.

8. Thilagavathy, K. P, Santha, A. 'The Achromatic and bChromatic Colouring of Central Graph of Book Graph and Shadow graph of Path graph' International Journal of pure and applied mathematics, 113,1-9,2017.

9. VernoldVivin, J.,Thilagavathi, K.'On Harmonious colouring of Central Graphs', Far East. Math. Sci (FJMS), 2,189-197, 2006. 